\title{
Modal passport of dynamically loaded structures: application to composite blades
}

\author{
Aleksey Mironov ${ }^{1}$, Deniss Mironovs ${ }^{2}$ \\ ${ }^{1}$ SIA D un D centrs, Riga, Latvia \\ ${ }^{2}$ Institute of Materials and Structures, Riga Technical University, Riga, Latvia \\ E-mail: 2deniss.mironovs@edu.rtu.lv (corresponding author)
}

\begin{abstract}
Close relation of modal properties to mechanical properties of structures presents interest for applied research and solutions of Structural Health Monitoring (SHM) tasks. The paper considers application of analytical and modal analysis techniques for structural research and monitoring of composite blades as well as required measurement tools. The Roving Hummer Technique (RHT) and Operational Modal Analysis OMA) were admitted as optimal methods for modal properties estimation, but 3-axial accelerometers and vibration deformation sensors - as most acceptable measurement tools. There is discussion of methodology and technical aspects required for modal properties estimation of static and rotating blades. Samples of composite blades preparation for measurements and equipment applied for testing are considered. Authors discuss the modal passport concept as the platform for applied studies of modal properties and for diagnostic purposes including Structural Health Monitoring (SHM) of operating structures. Typical modal passport structure is described as the set of modal parameters that are common for same type of blades and generalized dependences of modal parameters from operational factors. The paper discusses the principles of modal passport application for a particular blade based on its individual modal properties. There are conclusions about application of typical modal passport in industry.
\end{abstract}

Keywords: Modal analysis, rotating blades, modal passport.

\section{Introduction}

Mechanical properties of a structure determine its modal properties (mode shape, frequency and damping) that is the object of interest at the testing stage of a new structure, as well as at the operational stage of existing ones. Special attention in last years was paid to composite blades widely used in energy sector and aviation. Typically, the study of modal properties starts with a numerical simulation of blades, most often by means of standard software packages (Garinis, Dinulovic, \& Rasuo, 2012; Zaharia, 2015; Rabiei \& Galehdari, 2018). In some cases, alternative approaches are used in modeling, for instance Fuzzy Logic (Ganguli, 2001). Most often, an experimental study of blade modal properties are conducted using traditional techniques such as harmonic excitation or Roving Hammer Technique (Lakhdar, Mohammed, Boudjemâa, Rabiâ, \& Bachir, 2013). To measure the dynamic response of a blade Autonomous Wireless Sensor Network (Ramirez, Loendersloot, \& Tinga, 2013), built-in Accelerometer Arrays (White, Adams, \& Rumsey, 2011), Optical Fiber Bragg Grating and Classical Stretch Sensors (Luczak, 2010) may be used. Above techniques are required to determine modal parameters of a newly designed blade for wind power generator or a helicopter in order to verify computed models (White et al., 2011; Luczak, 2010). Sometimes the task is to check absence of resonances with periodic exciting loads or to verify the flutter margins. As OMA techniques evolve, it is getting possible to monitor the state of rotating blades (Agneni, Coppotelli, \& Grappasonni, 2010). To apply OMA for rotating blades a variety of accelerometers, strain gages, deformation sensors and in some cases digital images (Rizo-Patron \& Sirohi, 2017) are employed.

Based on the relationship between blades mechanical and modal properties any modification of blade mechanical state can be evaluated using multiple changes of its modal properties, including frequency, damping and shape of each mode. Typically, eigenvalues (frequency and damping) and eigenvector (shape) computed based on measured vibration data describe the modal properties of each mode. Aiming for blade monitoring we are faced with two problems. First, we need reliable technology providing measurements and determination of the modal properties of rotating blade, and second we need to differentiate the state modifications and effects of various operational factors on modal properties.

(C) 2019 Authors. Published by VGTU Press. This is an open-access article distributed under the terms of the Creative Commons Attribution (http://creativecommons.org/licenses/by/4.0/) License, which permits unrestricted use, distribution, and reproduction in any medium, provided the original author and source are credited. 


\section{Study of modal properties and impact of external factors}

To determine the modal properties of a fixed blade the techniques of Experimental Modal Analysis (EMA) are commonly used. For rotating blades, such methods are difficult to implement, therefore, OMA approach are getting widely spread (Debille, Pauwels, \& Peeters, 2005; Schwochow \& Jelicic, 2015; Peeters, De Troyer, Guillaume, \& Van der Auweraer, 2006; Böswald, Schwochow, Jelicic, \& Govers, 2017; Kerschen, 2013). These methods as signal processing procedures compute blade vibration modes using measurements in multiple Degrees of Freedom (DOF). OMA application requires to satisfy the main assumption about random nature of excitation. In reality, blade rotation causes significant periodic loads, which, along with aerodynamic loads, cause forced vibrations of blades. Superimposing the latter may affect the modal parameters of the blade that should be taken into account when applying OMA (Peeters, Manzato, \& Van der Auweraer, 2013). In some cases, it is necessary to suppress the periodic component of excitation in the vibration signal.

Another problem is the operation mode of rotating blades influences on its modal properties. The rotation speed determines the inertial and aerodynamic loads on the blade. The constant component of load affecting the blade also have an impact on its mechanical properties. Other external factors may also influence the parameters of natural vibrations. For example, the ambient temperature affects the mechanical properties of materials, and respectively, their modal properties. Aiming for SHM it is important to take into account the effect of operational parameters on modal parameters.

\section{Analytical approach}

A typical approach to assess the impact of external factors on blade modal properties is based on finite element modeling, which deals with modal properties of an idealized blade model. When applying the analytical approach the mechanical properties of the structure are simulated with its mass $\{M\}$, stiffness $\{K\}$ and damping $\{C\}$ in combination with the structural response (as represented by $u$ ):

$$
[M]\{\ddot{u}\}+[C]\{\dot{u}\}+[K]\{u\}=0 .
$$

The external impact can be shown by simulating the effect of these factors on modal parameters. For example, acting by constant centrifugal and aerodynamic loads on a blade, its modal parameters, like stiffness and damping, change. If we denote the alteration of blade properties under the influence of a constant load as $\Delta C$ and $\Delta K$, then the response of the structure $\Delta u$ will be derivable from the following formula

$$
[M]\{\ddot{u}+\Delta \ddot{u}\}+[C+\Delta C]\{\dot{u}+\Delta \dot{u}\}+[K+\Delta K]\{u+\Delta u\}=0 .
$$

For a generalized assessment of the impact on a virtual blade, such approach is appropriate. However, virtual blade model does not allow assessing, how the modal properties of the individual specimen modify, so the experimental methods are required. In the next section, we discuss in-situ testing of composite blade and its modal properties study using modal analysis techniques.

\section{Testing and modal properties of blades}

There are two stages of composite blades modal testing. At first stage, the blade is tested in a static position using EMA, and the second stage is OMA techniques application to rotating blades.

\section{Static testing}

Typical EMA approach based on computation of Frequency Response Functions (FRFs) allows testing the blade in static position. The set of FRF is calculated based on measured both excitation and response in multiple DOFs allocated around the blade. Depending on testing goals, excitation could be harmonic or impact. The testing procedure considers consequent actuation and measurements in DOFs according to geometric model of the blade. Then all FRFs for all DOFs are computed and after FRF curve fitting the matrixes of modal parameters are determined.

Harmonic excitation allows higher credibility of modal parameters provided using step-by-step frequency sweeping in defined frequency range. This approach is most appropriate; for instance, to determine modal damping, as sufficient energy for excitation of steady-state vibrations ensures a high accuracy in estimation. The measurement set-up, including the DOFs number and allocation, depends on the resolution requirements. At the same time, the method of harmonic excitation is quite cost-intensive, especially in terms of test duration.

Where accuracy of damping determination is not so important, the Roving Hammer Technique (RHT) is more appropriate for static tests. Using this approach, the transducer with fixed DOFs measures the responses of the single impact made by the hammer "roving" all DOFs consequently around the blade. The equipment required are the impact hammer having an embedded force sensor and one or more transducers providing DOFs measurement in three direc- 
tions. RHT requires significantly less time than the harmonic excitation technique. Figure 1 illustrates the typical measurement set-up for blade RHT; the hammer icons indicate DOFs (places and directions) of hits, and three colored arrows indicate measured DOFs of the transducers.

Miniature 3-axial accelerometers and vibration deformation sensors are used in discussed tests. Figure 2 shows samples of both sensor types application on the composite blades. Measuring the response simultaneously in three directions is an advantage of three-axial accelerometers (Figure 2a) that acceptable for medium and large blades. Being lightweight, even with a fastener and cable, they do not affect significantly on the modal properties of tested blades, if its mass is at least few kg. For blades with a lower mass, vibration deformation transducers (Figure 2b) are more acceptable.

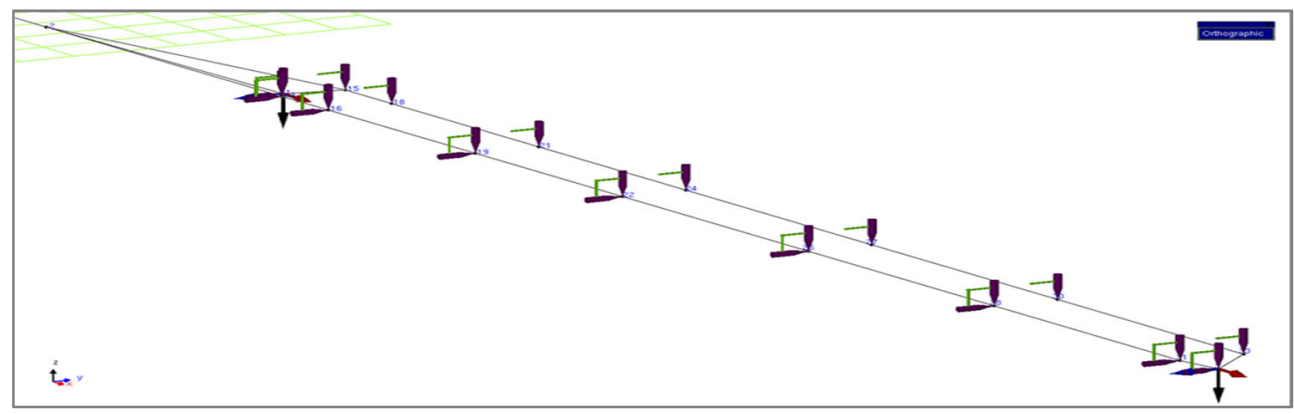

Figure 1. Measurement set-up for blade test with a hammer

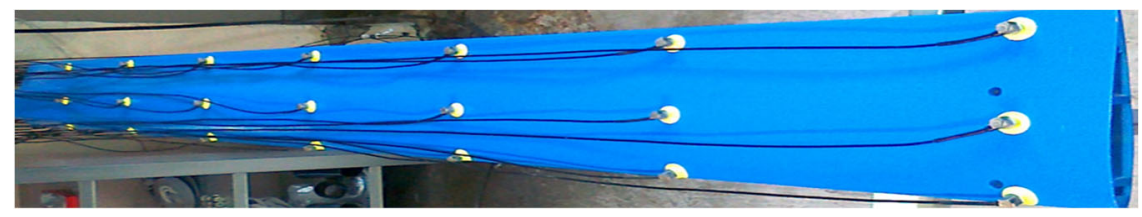

a)

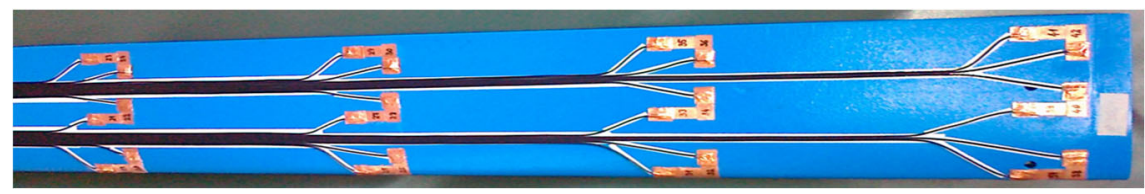

b)

Figure 2. Photo of vibration accelerometers (a) and vibration deformation sensors (b) on similar blades

Flexible films with piezo-electric layer are the basis of deformation transducers that generate an electric charge under extension or compression. Unlike conventional strain gages, such sensors do not require balancing as they respond to dynamic deformations only and are called vibration deformation transducers. Being almost weightless, they practically have no effect on modal properties of tested blade. The advantage of vibration deformation sensors is especially evident for rotating blades testing, where accelerometers may modify modal parameters of the blade and cause aerodynamic distortions. That is why vibration deformation sensors were chosen for testing rotating blades.

There are two stages of static tests of blade modal properties in discussed case. First, the RHT technique is applied for testing using 3-axial vibration transducers (accelerometers) and then software platform computes modes of blade vibrations. To verify modal properties the mode shape analysis and identification is required. For linear structure, such as blade, the diagram of FRF dependence on the relative deformation radius of the respective DOFs is a useful tool for mode shape identification. For convenience, colored lines relate FRF magnitude values along the blade axis. As an example, Figure 3a shows a diagram of mode shape for the third bending (flapping) mode, where blue line illustrates FRF magnitudes distribution along the front edge of the blade and the green line - along tailing edge. As deformation sensors characterize dynamic behavior of rotating blades it is necessary to define the compatibility between deflection and deformation shapes of the same mode. So, at the second stage of static tests, the modal parameters of the same blade are determined using the OMA techniques based on the signals of vibration deformation sensors.

The rubber hammer is used for blade excitation by blowing on the support of fixed blade with randomly varying force, direction and frequency. Then, using signals of deformation sensors, the modal parameters are computed using Artemis software platform in terms of the vibration deformation magnitudes. Based on the blade geometric model, which reflects the location of DOFs, Artemis provides an automatic processing of the measured response signals and resumes the set of modal parameters. 


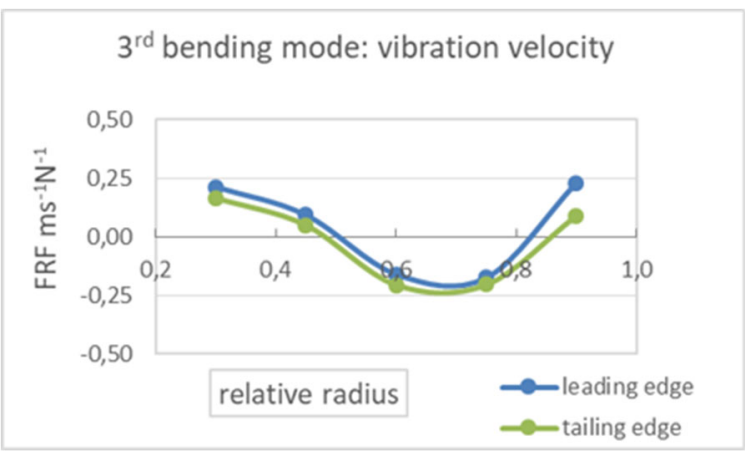

a)

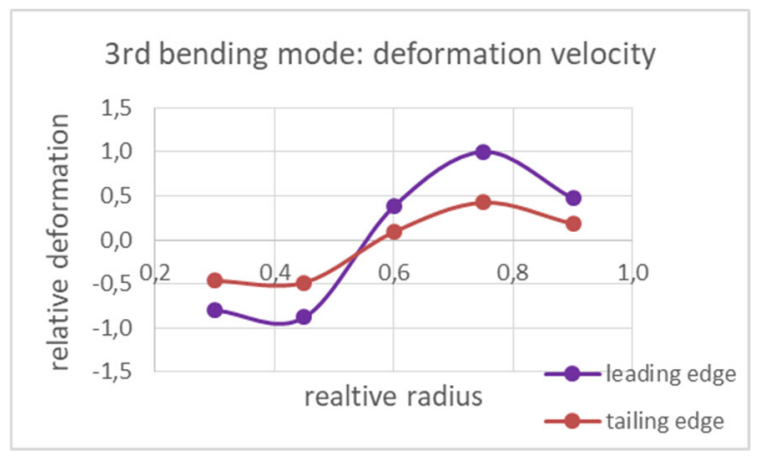

b)

Figure 3. Diagrams of vibration (a) and deformation (b) shapes of third flapping mode

Identification and classification of vibration modes is carried out based on the modal parameters obtained from both static tests. When identifying vibration and deformation modes, standard methods (such as MAC, COMAC, etc.) are not appropriate, since, even if they reflect the same mode, their curves are different. Therefore, an analytical approach shall be used for modes conformity and identification, concerning the nature of vibrations and deformations distribution along the length and chord of the blade for each vibration mode. Classifying the modes of composite blades typically bending (flapping and chord wise) as well as torsional modes are defined. Aiming for test results validation after initial modal identification the static tests to be repeated. The number of re-tests may vary depending on the tasks thereof. From plurality of modes detected in a given frequency range, the only modes selected for further consideration those have passed the test for repeatability. Identified and classified modes from static tests constitute a set of typical modes that will be further studied while testing the rotating blade.

\section{Rotating Blades Testing}

The technology for rotating blade testing includes methodical and measurement parts. The OMA techniques is methodological the basis but the advanced multichannel system measures and transmit signals of vibration deformation sensors. Figure 4 shows the measurement set-up of rotating blades with deformation sensors presented on Figure 2 and Figure 5 shows multichannel data acquisition unit (DAU) with wireless data transmission device.

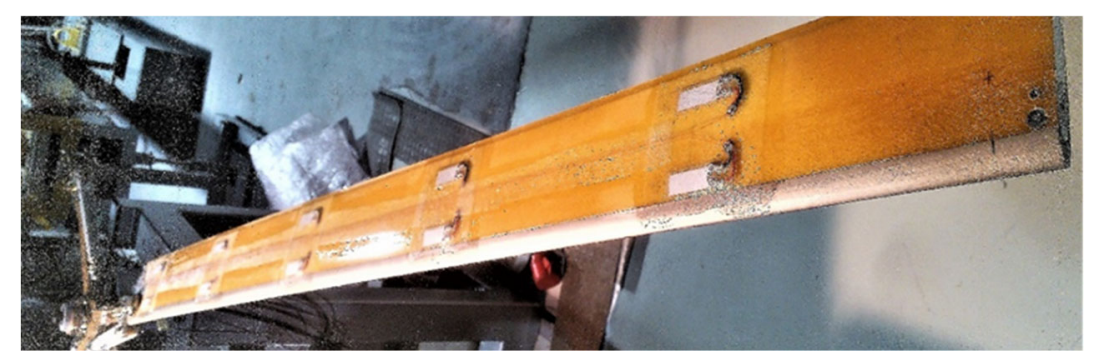

Figure 4. Embedded array of deformation sensors and wiring

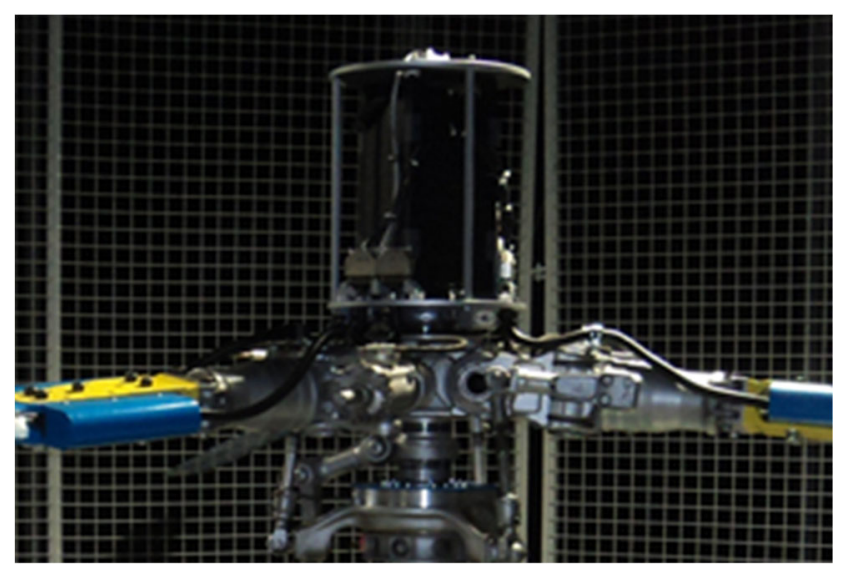

Figure 5. Multichannel DAU with connecting cables from blades 
In order to minimize the influence of sensors and their cables on the aerodynamic properties of the rotating blade, they are coated with the additional protective layer based on glass fiber veil and epoxy resin (Figure 4). Thin deformation sensors and its wiring (less than $1 \mathrm{~mm}$ ) allows the protective layer following the blade profile, excluding aerodynamic disturbances. The system providing data measurement and transmittance complies with the harsh environment under rotation speed of up to $1200 \mathrm{rpm}$. The wireless router rotating with DAU transmits the data to the workstation that ensures receiving, storing and further processing of data.

Modal properties of rotating blade depend of test conditions, like rotation speed and pitching. The loads affecting the blade in rotation is a sum of constant and variable components. Variable loads play useful role actuating vibrations and surface deformations used for determination of modes. Since blade vibrations affected by these loads remain elastics such loads practically does not affect the modal properties. Opposite, constant component of loads affect the mechanical properties of the blade, and, respectively, change its modal parameters. This effect has to be taken into account when testing rotating blades, since under the impact of centrifugal force and the constant lifting force, the stiffness of the blade structure changes, which in turn affects modal parameters.

Different factors determine operational mode of the rotor, so its influence to modal properties should be studied separately. For instance, to study rotational speed influence on blade modal properties a zero pitch of the blade to be arranged. This reduces lifting force and limits its influence on modal properties of rotating blade remaining the effect of centrifugal force only. To study the impact of lifting force on modal properties, the tests are performed at the same rotation frequency, altering the main rotor pitch.

\section{Modal Passport}

\section{Typical Modal Passport of a Blade}

Based on representative number of blades the set of typical vibration modes is determined using comparative analysis of vibration and deformation mode shapes. Such set of typical modes obtained name typical modal passport serves for complex description of typical properties of this blade type. The typical modal passport is valid within wide range of operating factors and becomes the basis for use of modal properties for testing and Structural Health Monitoring (SHM) of such type blades. Description of typical modal passport includes the matrixes of modal parameters: frequency, damping and shape. The composition of modes of a typical passport depends on the structure of a blade and includes the identified modes only that are common for all blades of the same type. Passport composition also depends of the task. When creating a typical passport for detuning a new blade from resonances; the number of modes is limited by order of the highest rotor-excited vibration harmonic, which the mode can cross in operational range. For diagnostic tasks, the resolution requirements determine the number of modes and the wider is the range of defects sought, the greater is the number of modes in a typical passport. A typical modal passport contains the values of the modal parameters of a typical, i.e. generalized blade. The values of modal parameters represent the expected means based on averaging of parameters for blade ensemble of the same type. In addition to averaged means, a typical modal passport contains also confidence intervals. Typical passport is multidimensional, as it describes dependence of modal parameters on different operational factors.

Typical modal passport serves as a template for individual modal passport of the particular blade. Data of this blade static test serves for individual passport computation. While the structure of individual passport conforms to typical one, each specific blade has its own values of modal parameters. Discrepancy of individual modal parameters in comparison to parameters of a typical passport does not exceed its confidence intervals.

In a practice, modal properties of composite blades vary significantly under the influence of external conditions. Being multidimensional, a modal passport is capable to characterize the impact of operational conditions on the modal parameters of a blade.

As an example, let us consider the dependence of blade modal parameters on the rotor speed, which has the most significant effect. Tests on the rotating blade are conducted in operational range from static to the maximal rotation speed that exceeds the nominal speed by $20-30 \%$. The modal parameters are computed separately for each rotor speed. As a result of data processing, parameters of vibration deformation modes are computed, including frequency, damping coefficient and surface deformation shape for each mode at a given rotation speed. Identifying deformation modes, only those would be considered that conform to the typical modal passport. The combination of modal parameters computed in all operational modes constructs an additional dimension of typical modal passport. This dimension reflects modal properties dependence of rotation frequency (rotation frequency dimension). Figure 6 graphically illustrates dimensions of modal passport.

The layer formed by the vertical axis (of modal parameters) and the sloping axis of mode number (15 modes in this sample) illustrates modal state of a blade in static position. The horizontal axis (of rotation speed) depicts an appropriate dimension of the typical passport. The layer crossing $40 \%$ rotation speed reflects modal parameters of the blade at this operational mode. The blue line connecting modal shape vectors between static state and $40 \%$ speed illustrates modal shape dependence on rotation speed. The number $N$ of tested modes of operation defines the dimension scale of this axis. When modal parameters of all typical modes for $N$ layers are computed the rotation speed dimension of typical modal passport is completed. Each layer of a typical passport has the same structure as the static 
one; however, parameter values of the same mode vary from page to page. For instance, the above-mentioned third flapping mode significantly changes its properties under the impact of centrifugal load. The modal frequency increases from $42 \mathrm{~Hz}$ in a static position to $64 \mathrm{~Hz}$ at the nominal rotation speed, and the damping coefficient drops from $4.1 \%$ to $0.6 \%$. At the same time, the modal deformation shape is modified as it is illustrated on Figure 7.

Another important factor influencing modal parameters is the pitch of a blade. In case of rotor testing with blade pitch variations, the modal passport gets a fourth dimension, which reflects the dependence of modal parameters on a pitch. For any other operational factors, like ambient temperature, flight speed, blade loading, etc. another dimensions of modal passport are formed basing on blade tests under according conditions.

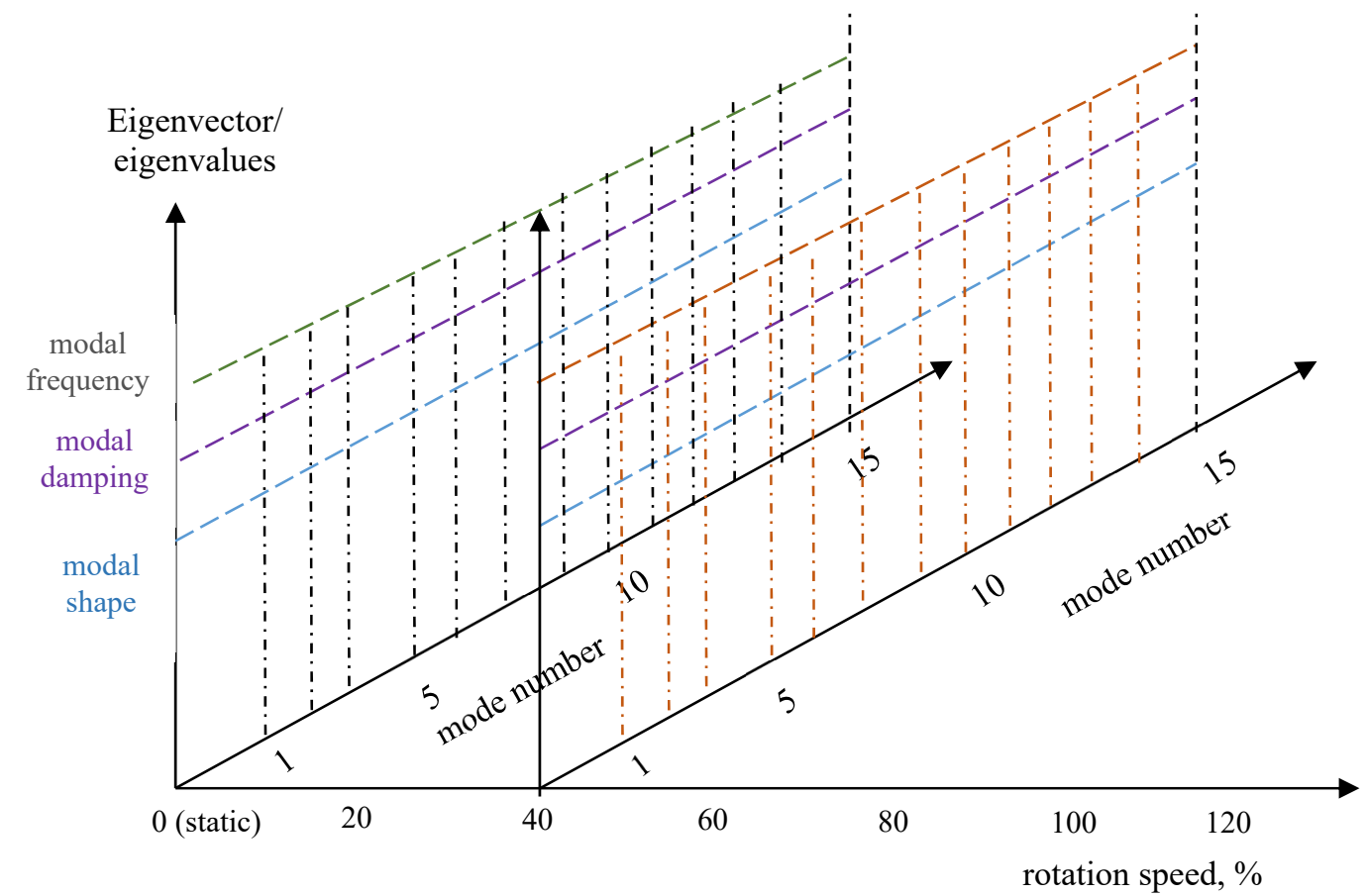

Figure 6. Graphic illustration of typical modal passport structure

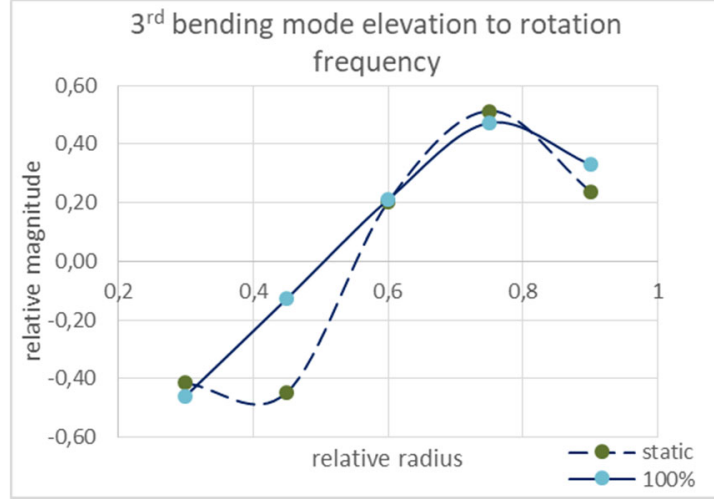

a)

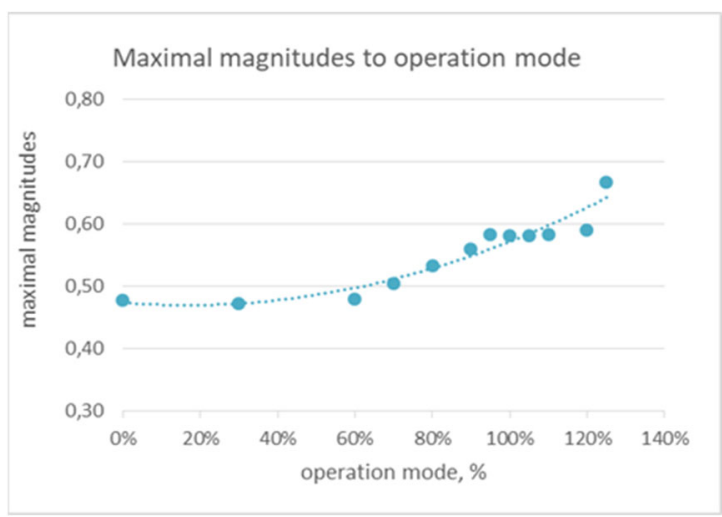

b)

Figure 7. Modal shape deformation with rotation speed growth (a); magnitude of $3^{\text {rd }}$ flapping mode in depend of operation mode (b)

For SHM purposes a typical passport requires another dimensions. For instance, an additional dimension should be added to relate modal parameters modifications in depend of defect scale (like delamination or other). Ultimately, the size of typical modal passport is not limited and can be increased by adding results of additional types of tests. The list of typical factors whose impact on modal properties of operating blade should be evaluated within preliminary study of operation conditions.

When the typical modal passport is built-up by all required data, the general dependences of modal parameters to operational factors could be defined. Figure $7 \mathrm{~b}$ illustrates the dependence of third flapping mode deformation magnitude to operational mode. Finally, the typical modal passport contains information on common modal properties of all blades of a given type and the generalized dependences of modal properties on typical set of operational factors. 
Creating a typical modal passport is a costly task, however once it has been done, it allows easy assessing and predicting the modal properties of any specific blade of this type. Contrary to typical passport the individual modal passport of any specific blade needs the basic layer only that is formed by testing in static state under standard conditions. When individual modal passport of specific blade has basic layer then its modal properties can be predicted for any operational conditions within the typical range of operating factors. Such prediction is computed applying generalized dependences of modal properties (from typical passport) to modal parameters of basic layer of individual passport. Using such approach the modal properties defined from the blade at any operational condition is recalculated to typical conditions. Providing such opportunities modal passport becomes the platform for diagnostics of blades in industrial conditions and also SHM of operating blades in energy (wind generators) or aviation (helicopters).

\section{Conclusions}

The platform of modal passport simplifies different research and industrial procedures related to modal analysis as illustrated on the results of composite blade testing. First, it allows identification of vibration/deformation modes of individual blade using data independent of test or operation conditions. For instance, data measured in winter or summer, from fixed or rotating blades, maximally loaded or in idle mode could be used. Secondly, to predict modification of individual blade properties in depend on some operational factor someone does not need to study behavior of this blade. It is enough to have only its modal parameters in static state and then to use the generalized dependence of typical passport on above operational factor. For instance, potential resonance can be checked knowing modal frequency in the static test and using the dependence of modal frequency (of a typical passport) on the rotational speed.

In terms of diagnostics (SHM) it is important to predict condition modification of a particular operating blade using just the modal parameters of the static test. Based on the dependencies of the typical passport a prediction can be made independently on operational mode and without respective tests of that blade. It means that for an individual blade its modal properties can be simulated for any combination of typical operating factors. The simulated blade properties are characterized by both the predicted value and confidence intervals. If some modal parameter of a particular blade exceeds the intervals, it indicates that its modal and mechanic properties exceed the typical ones.

\section{Acknowledgement}

The paper uses materials related to research No. 4 "Demonstrator of Aircraft Perspective Vibration Diagnostic Technology Complex" inside project No.1.2.1.1/18/A/001 within the Energy and Transport Competence Center in cooperation with Central Finance and Contracting Agency of Latvia. This work contains the results of the research study and development of vibration diagnostic techniques demonstrator.

\section{References}

Agneni, A., Coppotelli, G., \& Grappasonni, C. (2010). Operational modal analysis of a rotating helicopter blade. Proceedings of ISMA2010 including USD2010. Operational Modal Analysis (pp. 3249-3262).

Böswald, M., Schwochow, J., Jelicic, G., \& Govers, Y. (2017, May). New concepts for ground and flight vibration testing of aircraft based on output-only modal analysis. In IOMAC $2017-7^{\text {th }}$ International Operational Modal Analysis Conference. Ingolstadt, Germany.

Debille, J., Pauwels, S., \& Peeters, B. (2005). The benefits of operational modal analysis of aircraft and spacecraft structures. ETTC 2005 - European Test \& Telemetry Conference. Belgium.

Ganguli, R. (2001). A fuzzy logic system for ground based structural health monitoring of a helicopter rotor using modal data. Journal of Intelligent Material Systems and Structures, 12(6), 397-407. https://doi.org/10.1106/104538902022598

Garinis, D., Dinulovic, M., \& Rasuo, B. (2012). Dynamic analysis of modified composite helicopter blade. FME Transactions, 40(2), 63-68.

Kerschen, G. (2013). Nonlinear modal analysis of a full-scale aircraft. Journal of Aircraft, 50(5), 1409-1419. https://doi.org/10.2514/1.C031918

Lakhdar, M., Mohammed, D., Boudjemâa, L., Rabiâ, A., \& Bachir, M. (2013). Damages detection in a composite structure by vibration analysis. Energy Procedia, 36, 888-897. https://doi.org/10.1016/j.egypro.2013.07.102

Luczak, M., Peeters, B., \& Dziedziech, K. (2010). Static and dynamic testing of the full scale helicopter rotor blades. Proceedings of ISMA 2010 including USD2010. Lightweight Panels and Structures (pp. 2131-2143).

Peeters, B., De Troyer, T., Guillaume, P., \& Van der Auweraer, H. (2006). In-flight modal analysis - a comparison between sweep and turbulence excitation. In Proceedings of the ISMA 2006 International Conference on Noise and Vibration Engineering. Leuven, Belgium.

Peeters, B., Manzato, S., \& Van der Auweraer, H. (2013). Solutions to deal with harmonics and noise for helicopter in-flight data dynamic identification. In $5^{\text {th }}$ International Operational Modal Analysis Conference. Guimarães, Portugal.

Rabiei, H., \& Galehdari, S. A. (2018). Modal numerical analysis of helicopter rotor sample using Holzer-Myklestad method. Journal of Stress Analysis, 3(1), 111-121. 
Rizo-Patron, S., \& Sirohi, J. (2017). Operational modal analysis of a helicopter rotor blade using digital image correlation. Experimental Mechanics, 57(3), 367-375. https://doi.org/10.1007/s11340-016-0230-6

Sanchez Ramirez, A., Loendersloot, R., \& Tinga, T. (2013). Helicopter rotor blade monitoring using autonomous wireless sensor network. In B. Basu (Ed.), Proceedings of the $10^{\text {th }}$ International Conference on Condition Monitoring and Machinery Failure Prevention Technologies - CM/MFPT 2013 (pp. 775-782). Krakow, Poland: The British Institute of Non-Destructive Testing.

Schwochow, J., \& Jelicic, G. (2015). Automatic operational modal analysis for aeroelastic applications. In $6^{\text {th }}$ International Operational Modal Analysis Conference 2015. Gijón, Spain.

White, J. R., Adams, D. E., \& Rumsey, M. A. (2011). Modal analysis of CX-100 rotor blade and Micon 65/13 wind turbine. In T. Proulx (Ed.), Structural dynamics and renewable energy (vol. 1, pp. 15-27). Conference Proceedings of the Society for Experimental Mechanics Series. Springer, New York, NY. https://doi.org/10.1007/978-1-4419-9716-6 2

Zaharia, S. M. (2015). The modal analysis of a carbon fiber helicopter blade. Journal of Industrial Design and Engineering Graphics, 10(2), 23-26. 